\title{
Dynamics of nonstationary dipole vortices
}

\author{
J. S. Hesthaven, J. P. Lynov, and J. Nycander ${ }^{a}$ ) \\ Association EURATOM-Risø National Laboratory, Optics and Fluid Dynamics Department, \\ P. O. Box 49, DK-4000 Roskilde, Denmark
}

(Received 22 January 1992; accepted 6 November 1992)

\begin{abstract}
The dynamics of tilted dipole vortices in the equivalent barotropic vorticity (or Hasegawa-Mima) equation is studied. A recent theory is compared with numerical simulations and found to describe the short time behavior of dipole vortices well. In the long time limit the dipoles are found to either disintegrate or relax toward a steady eastward propagating dipole vortex. This relaxation is a consequence of nonviscous enstrophy loss by the dipole vortex.
\end{abstract}

\section{INTRODUCTION}

The existence of steadily propagating dipole vortex solutions to the Euler equation was first noted by Lamb. ${ }^{1}$ Later Stern ${ }^{2}$ showed the existence of a similar solution (which he labeled "modon") on the $\beta$ plane, and Larichev and Reznik ${ }^{3}$ extended it to the equivalent barotropic vorticity equation. ${ }^{4}$ Since then dipole vortices have been the subject of a large number of studies. We note in particular that they appear naturally in various laboratory experiments, for instance in a turbulent wake field in a thin soap film, ${ }^{5}$ or after injecting a jet into a rotating fluid. ${ }^{6}$ The westward propagating dipole vortex has also been proposed by McWilliams ${ }^{7}$ as a model of atmospheric blocking. However, as will be seen below, this dipole vortex is unstable, implying that it is unlikely to be the cause of blocking.

The same equation as the equivalent barotropic vorticity equation can be derived for nonlinear drift waves in a magnetized plasma with a density gradient. ${ }^{8}$ In the plasma physics community the equation is known as the "Hasegawa-Mima equation." It has, in the last decade, been realized that exact dipole vortex solutions exist in a large class of nonlinear equations, which describe various types of plasma motion. Since dipole vortices have the ability of carrying trapped particles over long distances, they may be important for understanding anomalous transport across the magnetic flux surfaces, corresponding to transport in the north-south direction in geophysical flows.

In the presence of the $\beta$ effect, there are two different kinds of stationary dipole vortices: those propagating westward, i.e., in the same direction as, but faster than the linear Rossby waves, and those propagating eastward. The numerical solutions by Makino et al. ${ }^{9}$ who studied tilted dipole vortices (i.e., launched with some angle to the eastwest axis), revealed an important difference between the two classes. While the trajectory of the tilted eastward propagating vortices was gently oscillatory, the westward propagating ones executed a cycloid motion with large excursions from the original latitude. The latter case was, however, just mentioned in passing by the authors, and

\footnotetext{
ajPermanent address: Department of Technology, Uppsala University,
} Box 534, S-751 21 Uppsala, Sweden. they did not draw attention to the fact that it implies that the steady westward propagating dipole is unstable.

Verkley ${ }^{10}$ obtained stationary dipole vortices in a uniform westerly flow on a sphere, and found that they are unstable by solving the linear eigenvalue problem numerically. Since these vortices are in resonance with the linear waves, his solutions contain a global pattern of standing Rossby-Haurwitz waves, and are rather different from the localized dipole vortices studied here.

Zabusky and McWilliams ${ }^{11}$ proposed to model dipole vortices by two point vortices with different sign, and studied this system for the eastward, oscillating case. This point vortex model exhibits the same qualitative difference between eastward and westward propagation as the numerical simulations, and predicts that the westward propagating dipole is unstable, as was pointed out by Hobson. ${ }^{12}$ The existence of this instability has been confirmed by direct numerical simulations. ${ }^{12,13}$

Recently, a general equation of motion for dipole vortices was proposed by Nycander and Isichenko. ${ }^{14}$ It is related to the point vortex model, but the dynamics is somewhat different, and the coefficients of the equation are defined directly as integrals over a dipole vortex with arbitrary smooth profile.

In the present work the motion of dipole vortices propagating in arbitrary directions will be studied by direct numerical simulations. In Sec. II the basic equation and the Nycander-Isichenko ${ }^{14}$ theory is reviewed. Section III deals with the evaluation of the theory and the study of the long time behavior of the vortices. Since tilted dipole vortices are not exact steady solutions, they gradually lose energy and enstrophy to the surrounding fluid. The effect of this on the motion will be the subject of Sec. IV. In Sec. $\mathrm{V}$ a brief conclusion is given.

\section{THEORY}

The equivalent barotropic vorticity equation (or the Hasegawa-Mima equation) is

$$
\frac{\partial}{\partial t}\left(\phi-\nabla^{2} \phi\right)-\beta \frac{\partial \phi}{\partial x}-\left[\phi, \nabla^{2} \phi\right]=0,
$$

where $\phi$ represents the perturbation of the fluid depth, [ , ] the Jacobian, and $\beta$ is proportional to the gradient of the 
Coriolis parameter. The maximum phase velocity of linear Rossby waves propagating in the $x$ direction is $-\beta$ in dimensionless units. Note that in plasma physics the variation of the density is usually assumed to be in the $x$ direction, whereas here it is taken to be in the $y$ direction. The $x$ axis is pointing east and the $y$ axis north.

It is straightforward to show that on an infinite domain Eq. (1) conserves energy,

$$
E=\frac{1}{2} \int\left[\phi^{2}+(\nabla \phi)^{2}\right] d x d y
$$

and enstrophy (or pseudomomentum, in the terminology of McIntyre and Shepherd ${ }^{15}$ ),

$$
\Omega=\frac{1}{2} \int\left(\phi-\nabla^{2} \phi\right)^{2} d x d y
$$

The potential vorticity is a Lagrangian invariant

$$
\Gamma=\nabla^{2} \phi-\phi+\beta y,
$$

being conserved for each individual fluid element, moving with the velocity $\mathrm{v}=\hat{z} \times \nabla \phi$.

In 1976, Larichev and Reznik ${ }^{3}$ found an exact dipole vortex solution to Eq. (1);

$$
\begin{aligned}
& \phi(r, \theta)=U \frac{\rho^{2}}{\kappa^{2}}\left[a \frac{J_{1}(\kappa r)}{J_{1}(\kappa a)}-r\left(\frac{\kappa^{2}}{\rho^{2}}+1\right)\right] \sin \theta \quad r<a, \\
& \phi(r, \theta)=-U a \frac{K_{1}(\rho r)}{K_{1}(\rho a)} \sin \theta, \quad r>a,
\end{aligned}
$$

where

$$
\rho^{2}=\frac{U+\beta}{U} .
$$

Here $\rho^{2}>0$ is necessary to ensure that the solution in Eq. (5) is localized, i.e., $U<-\beta$ or $U>0$. Thus the dipole and the linear waves cover complementary parts of the velocity space. Also $J_{1}$ and $K_{1}$ are the first-order Bessel and modified Bessel function, respectively, $a$ is the radius of the circular separatrix, and $U$ is the propagation velocity; $\kappa$ is found as the first solution to the equation

$$
\frac{K_{2}(\rho a)}{\rho a K_{1}(\rho a)}=-\frac{J_{2}(\kappa a)}{\kappa a J_{1}(\kappa a)},
$$

ensuring continuity of $\nabla \phi$ at $r=a$, and $\phi$ is continuous up to second derivatives, while higher-order derivatives are discontinuous at $r=a$.

It is seen from Eqs. (5)-(7) that for $U<-\beta, \rho^{2}<1$ and for $U>0, \rho^{2}>1$ so that the westward propagating dipole vortex is smoother and less localized than the eastward propagating dipole vortex. For $|U|>\beta, \rho^{2} \simeq 1$ and the dipole shape becomes independent of the direction of propagation.

In this paper, the focus with be on dipole vortices launched at different angles, $\alpha$, with respect to the $x$ axis. The main dynamics of such dipoles, as explained qualitatively by Makino et al., ${ }^{9}$ may be understood from the conservation of potential vorticity in the trapped fluid inside the separatrix. When the dipole starts propagating-say, to the northeast--the vorticity of the dipole halves must decrease according to Eq. (4), so that the cyclone (situated northward) becomes weaker and the anticyclone stronger. The dipole therefore swings around southward and starts increasing the vorticity and eventually reaches the original latitude. The southeastward motion continues until the cyclonic part of the dipole becomes so strong that the dipole swings around and starts propagating to the northeast again. As a result we obtain an oscillatory motion. By using similar arguments it is easy to realize that a westward propagating dipole is unstable to small perturbations. ${ }^{12,13}$

By quantifying these arguments, Nycander and Isichenko ${ }^{14}$ recently derived a simple and approximate equation of motion for the velocity $\mathbf{U}$ of dipoles propagating in arbitrary directions:

$$
\frac{d \mathrm{U}}{d t}=\mathbf{U} \times \hat{z} y \beta U \frac{S}{P_{d}},
$$

where $S=\pi a^{2}$ is the area of the dipole inside the separatrix, $y$ is the $y$ coordinate of the center of the dipole vortex, and $\boldsymbol{P}_{d}=\left|\mathbf{P}_{d}\right|$ is the magnitude of the dipole moment,

$$
\mathbf{P}_{d}=\int\left(\phi-\nabla^{2} \phi\right) \mathbf{r} d x d y,
$$

with $\mathbf{r}=0$ in the center of the dipole vortex. Equation (8) is obtained by noting that the radius of curvature of the trajectory is equal to the ratio between the dipole moment and the monopole moment (i.e., the integral of $\phi-\nabla^{2} \phi$ ) of the dipole vortex, and also that the monopole moment varies linearly with the $y$ coordinate of the vortex. The latter is a consequence of the conservation of potential vorticity inside the separatrix. It is assumed that the internal structure of the dipole remains unchanged, implying that $S$ and $P_{d}$ are constant, and that the radius of curvature of the trajectory is much larger than the diameter of the dipole. This is justified if $\beta / U<1$.

Equation (8) describes similar (but not identical) dynamics as the point vortex model. ${ }^{11,12,16}$ Note, however, that the coefficients of Eq. (8) are directly defined by the properties of a dipole with an arbitrary smooth profile, while the connection between the parameters of the point vortex model and a smooth dipole vortex is less clear.

Equation (8) may be integrated, and the oscillation frequency, $\omega$, obtained,

$$
\omega=U \frac{\pi}{2}\left(\frac{\beta S}{P_{d}}\right)^{1 / 2} \frac{1}{K(k)},
$$

where $K$ is a complete elliptic integral of the first kind with the argument $k=|\sin (\alpha / 2)|$, where $\alpha$ is the maximum angle between the trajectory and the $x$ axis. Here $\alpha=0$ for an exactly eastward propagating steady dipole.

The maximum displacement in the north-south direction, $y_{t}$, is found to be

$$
y_{t}= \pm\left(P_{d} / \beta S\right)^{1 / 2} 2 \sin (\alpha / 2),
$$

and the displacement along the east-west direction, $L_{w}$, during one full oscillation, here referred to as the oscillation length, is 


$$
L_{w}=4\left(P_{d} / \beta S\right)^{1 / 2}[2 E(k, \pi / 2)-K(k)],
$$

where $E$ is an elliptic integral of the second kind.

This theory assumes that the dipole vortex does not lose any of its enstrophy to the surrounding fluid. However, Nycander and Isichenko ${ }^{14}$ identified two possible mechanisms of such a loss. The first one is caused by the outer fluid elements being displaced in the north-south direction by the vortex as it passes by. Because of the conservation of potential vorticity they then acquire some amplitude of $\phi-\nabla^{2} \phi$. The second mechanism is driven by the pulsation of the two dipole halves, causing fluid elements to be trapped and untrapped regularly. Fluid elements that are released at a different meridional position than where they were trapped then acquire some amplitude of $\phi$ $-\nabla^{2} \phi$. Consequences of this enstrophy loss will be discussed further in Sec. IV.

\section{NUMERICAL RESULTS}

The dynamic behavior of tilted dipoles has been investigated by numerical solution of Eq. (1). The code is based on a pseudospectral spatial approximation scheme in a periodic domain of size $\left(L_{x}, L_{y}\right)=(15,15)$ with $(M, N)$ $=(128,128)$ modes. All simulations are done with a dipole diameter of 2 , corresponding to about 17 wavelengths per dipole diameter. A simple measure of the fidelity of the code is the ability of reproducing the speed of a translating dipole. It has been found that with seven wavelengths per dipole diameter the speed is reproduced with an error less than 5\%. Similar demands for spatial resolution were also found by Carnevale et al. ${ }^{17}$ With 17 wavelengths per dipole diameter, as in our simulations, the error is less than $0.5 \%$.

A third-order Adams-Bashforth predictor-corrector method is used as time integration and a zero-padding scheme is used to remove aliasing errors. As initial value is used the exact solution in Eq. (5) rotated $\alpha$ degrees. The simulations were done on an IBM 3090/VF, situated at the Technical University of Denmark, with a typical running time for each time unit in Eq. (1) of $8 \mathrm{CPU}$ sec with 20 time steps and $86^{2}$ active modes.

\section{A. Cutting facility}

Before the detailed presentation of the numerical results, a problem will be addressed which is caused by the periodic boundary conditions used in the numerical calculations. A nonstationary propagating dipole loses energy and enstrophy to a wake field, which finally evolves into linear waves propagating westward. Because of the periodic boundary conditions the dipole and the linear waves eventually collide and some interaction will occur. It was observed that this may have a strong impact on the behavior of the dipole. This problem has been noticed previously by Makino et al., ${ }^{9}$ although no solution was found.

Since the linear waves mainly consist of long wavelength waves, viscosity has only a marginal effect on them.

The technique implemented here is to multiply $\phi$ by a cutting function at regular time intervals. The cutting function is defined as

$$
f_{\text {cut }}(r)=\left\{\begin{array}{l}
1, \quad \text { if } r<r_{\text {cut }}, \\
\exp \left(-\frac{\left(r-r_{\text {cut }}\right)^{2}}{\sigma}\right),
\end{array}\right.
$$

with $r=0$ at the center of the dipole structure; $r_{\text {cut }}$ must be chosen large enough to ensure that the dynamics of the dipole vortex is not significantly affected. The Gaussian truncation is used to avoid steep gradients, which may introduce numerical ringing.

In order to fully remove the linear waves the truncation must be done with a maximum time interval of

$$
t_{\text {cut }}<\frac{L_{x}-2 r_{\text {cut }}}{U+\beta},
$$

where $L_{x}$ is the length of the periodic domain in the eastwest direction.

The two constants $r_{\text {cut }}$ and $\sigma$ are chosen empirically, using the rugged invariants energy and enstrophy as diagnostics. For an eastward propagating steady dipole with initial parameters $U=0.1, a=1.0, \beta=0.05$, the temporal behavior of the invariants has been studied with a parameter scan in $r_{\text {cut }}(=2.5 a, 3.0 a, 3.5 a, 4.0 a)$ and $\sigma(=1.0,2.0$, 4.0 ). To ensure an insignificant effect of ringing, under the constraint of a minimal spatial extension of the total cutting function, the optimum choice of parameters was found to be $r_{\text {cut }}=3.5 a, \sigma=1.0$. This set of parameters will be used in all simulations unless otherwise stated.

Repeated cutting decreases the energy of a steady dipole vortex by approximately $1 \%$ and the enstrophy by $8 \%$, i.e., the effect of the cutting is to smoothen the structure. It is noteworthy that the dipole vortex responds to the cutting by becoming more localized, which is done by slightly decreasing the propagation velocity $U$. After some time the energy and enstrophy then stop decreasing, although the cutting continues.

As a final test of the cutting scheme, the trajectory of an oscillating dipole has been compared with the trajectory of a similar dipole simulated in an elongated box without cutting. Only the horizontal dimension was expanded, since the dipole vortex in average propagates in this direction. The trajectory is defined as the motion of the center of the dipole vortex. The center is found by doing a twodimensional (2-D) Taylor expansion around the position of minimum and maximum $\phi$ in order to avoid problems with the discrete configuration space, and then determining the point halfway between the two. The result is shown in Fig. 1. Until the time shown by the vertical dashed line, the dipole vortex in the elongated box has not yet had time to interact with the linear waves. The trajectory obtained with the cutting facility applied in the smaller box shows good conformity with the trajectory obtained in the elongated box. We conclude that the cutting scheme does not affect the dynamics significantly, and facilitates the study of long time behavior of dipole vortices. The trajectory obtained from a simulation in a small box without the cutting illustrates the need for the cutting facility.

As an additional check we also repeated the simulation in a small box with double resolution. The trajectory was then indistinguishable by eye from the one shown in Fig. 1. 


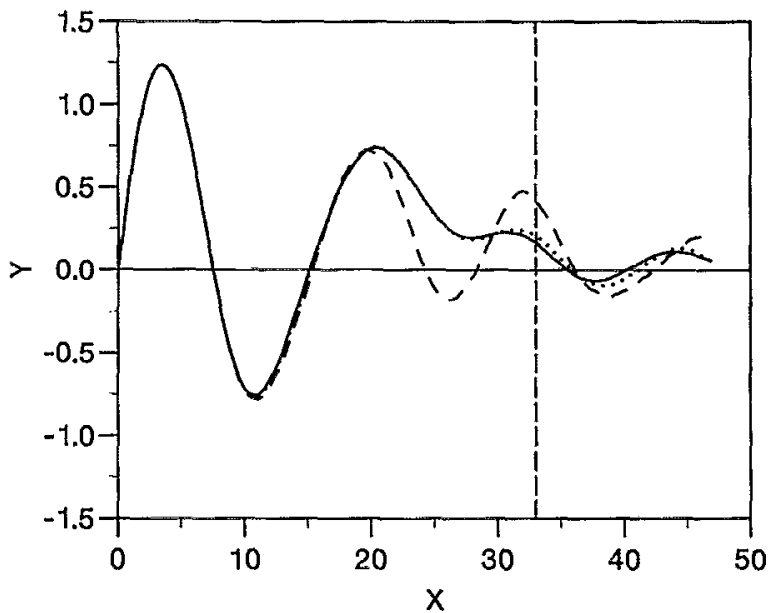

FIG. 1. Trajectories of dipoles illustrating the effect of the cutting scheme. Initial parameters were $U=0.1, a=1.0, \beta=0.05, \alpha=30^{\circ}$. The solid line is the trajectory obtained in the elongated box $(M=512$, $L_{x}=60$ ) and the dotted line is the trajectory obtained in a smaller box with the cutting applied. The dashed line is the trajectory obtained in a small box without cutting, illustrating the need for this scheme.

\section{B. Short time behavior}

Simulations of oscillating dipoles have been performed with $a=1.0, U=0.1, r_{\text {cut }}=4.0 a$ in the cutting function and $0.01<\beta<0.35$. The reason for choosing $r_{\text {cut }}$ slightly bigger than found in Sec. III A is that the dipole becomes slightly less localized when $\beta$ decreases (the optimum choice of $r_{\text {cut }}=3.5 a$ was found for $\beta=0.05$ ).

In most simulations no viscosity was included in Eq. (1), but a few test runs were performed with a hyperviscosity term proportional to $\nabla^{6} \phi$ and large enough to remove ripples in the vorticity field. The results were found to be almost unaltered by this dissipative term.

Simulations were performed to test the assumption of constant dipole moment by applying the cutting shortly after a quarter of a period, and calculating the dipole moment. The dipole moment was found to change less than $0.5 \%$ for different values of $\beta$ and $\alpha$.

To test Eqs. (10)-(12), the dipole behavior was simulated for different values of $\beta$ and $\alpha$. The three characteristic parameters $\omega, y_{t}, L_{w}$ were determined at the first maximum of the oscillation-after a quarter of a period. The maximum was determined by making a parabolic fit to the center point and the two surrounding points. The estimated parameters were then found by extrapolation to a whole period. The reason for using only a quarter of a period is that the theory above assumes negligible loss of energy and enstrophy, which may best be approximated by only analyzing the short time behavior.

In Fig. 2, the simulated oscillation frequency is compared with Eq. (10). We observe good agreement between theory and simulations for $\beta / U<1$ except for $\alpha \leqslant 30^{\circ}$. In Fig. 3 the maximum amplitude of the oscillation is plotted and compared with the estimate from Eq. (11), and Fig. 4 shows the comparison between simulations and the oscillation length from Eq. (12). In the limit $\beta / U<1$, the fig-

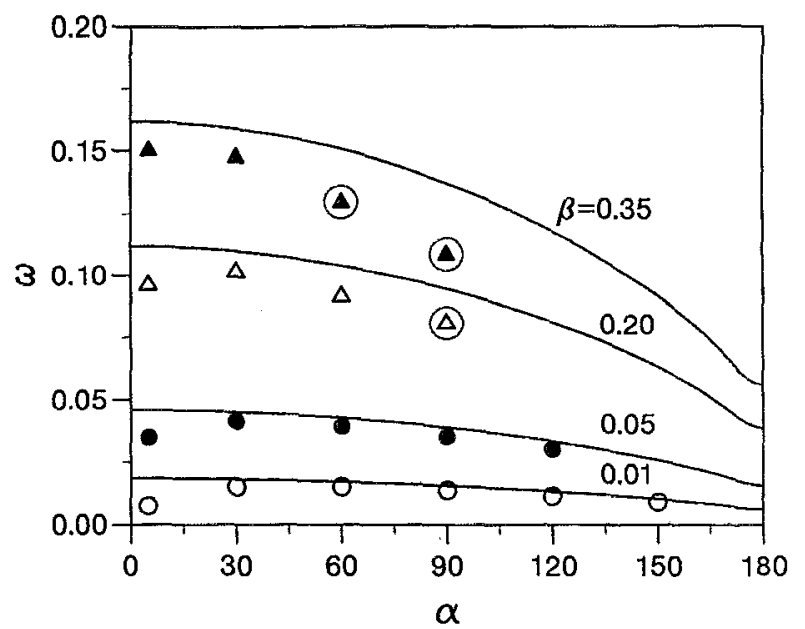

FIG. 2. Oscillation frequency, $\omega$, obtained from Eq. (10) compared with computed results. $O: \beta=0.01, \quad: \beta=0.05, \Delta: \beta=0.20, \Delta: \beta=0.35$. Disintegrating dipole vortices are encircled. Here and in all subsequent figures $a=1.0$ and $U=0.1$.

ures show good correspondence between simulations and theory even for a very large initial angle. However, there is some unexpected deviation for small angles. This will be discussed below.

In the region $\beta / U>1$, the qualitative dependence of the oscillation frequency, $\omega$, on the angle is correct, but the exact value is seen to be too low. This may be explained by energy loss causing the dipole vortex to decrease its propagation velocity. The good agreement for $y_{t}$ and $L_{w}$ supports this assumption.

Some of the dipoles disintegrate shortly after a quarter of a period, as marked in Figs. 2-4. The large deviation for $\beta / U>1$ and large initial propagation angle are most likely connected to processes causing the disintegration.

In order to investigate the rather unexpected deviation between the numerical results and the theory in the limit of

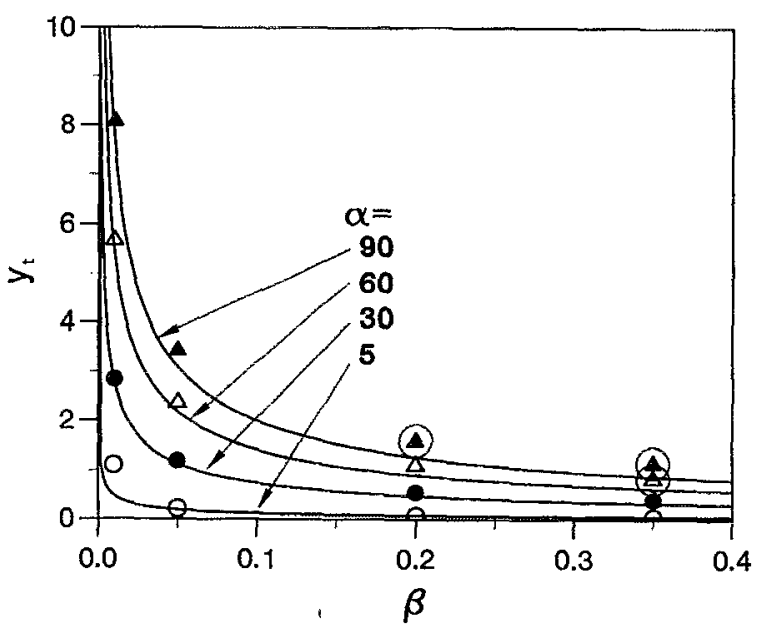

FIG. 3. Maximum vertical displacement, $y_{b}$ obtained from Eq. (11) compared with computed results. $\bigcirc: \alpha=5^{\circ}, \quad: \alpha=30^{\circ}, \Delta: \alpha=60^{\circ}, \Delta: \alpha$ $=90^{\circ}$. Disintegrating dipole vortices are encircled. 


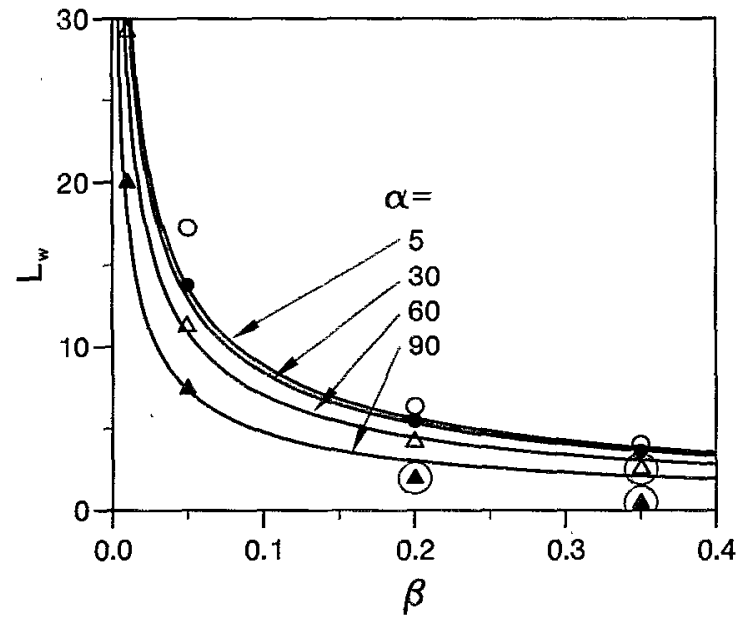

FIG. 4. Oscillation length, $L_{w}$, obtained from Eq. (12) compared with computed results. Symbols as in Fig. 3.

$\beta / U<1$ and $\alpha<90^{\circ}$ a detailed study of this region has been done. The result is shown in Fig. 5. The deviation is not an effect of the cutting, since for $\beta=0.05$ the dipole vortex propagates more than a quarter of an oscillation before being perturbed by the cutting.

One may speculate that the deviation is caused by some internal transient process. It has been observed from scatter plots that the internal reorganizing process has a stronger effect when $\alpha$ is small, causing the functional dependency of $\Gamma$ on $\phi+U y$ inside the separatrix to become weakly nonlinear after a quarter of an oscillation. This idea is supported by the fact that calculating the oscillation frequency of the dipole after several oscillations yields increased agreement with the theory as shown in Fig. 5.

As has been shown, the above theory seems to agree well within the given limits. However, as will become evident in the next section, the energy and enstrophy losses have a strong impact on the long time behavior of the dipole, implying that the theory is only valid for the first

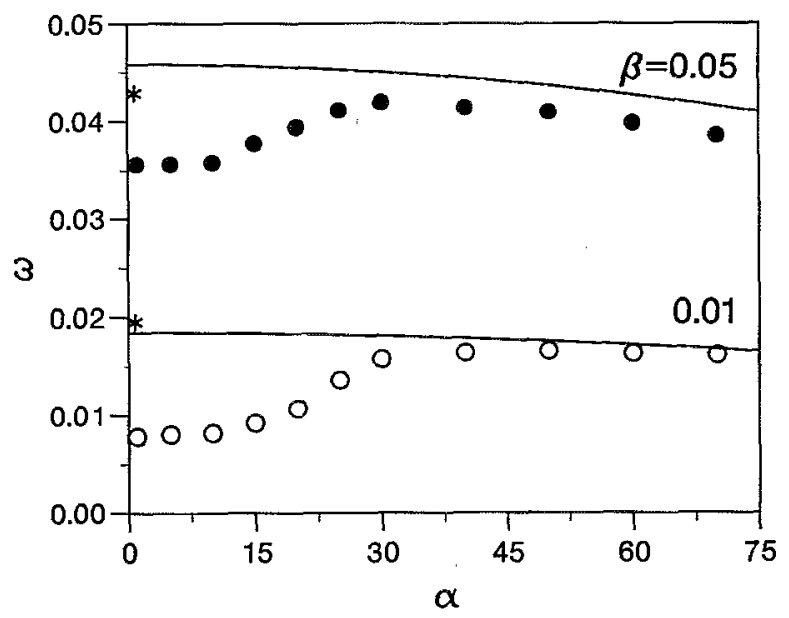

FIG. 5. Magnification of Fig. 2. * mark the results obtained after several oscillations.

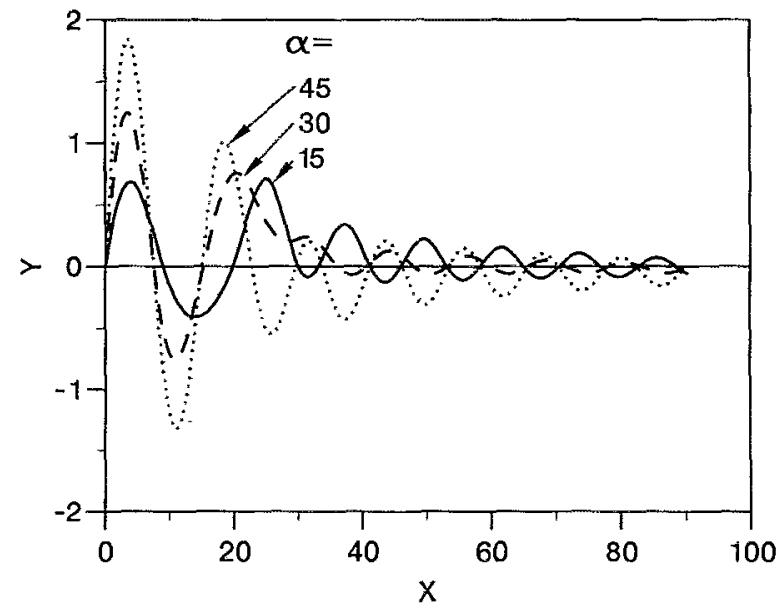

FIG. 6. Trajectories for initially eastward propagating dipole vortices, i.e., $U$ in Eq. (5) is positive; $\beta=0.05$.

few oscillations. After these the dipole vortex has changed significantly, and the theory fails to describe the trajectory of the oscillating dipole based on the initial conditions.

\section{Long time behavior}

After having studied the short time dynamics of tilted dipoles, which, at least in part of the parameter space, seems to be strongly affected by initial transient effects, the focus will now be on the behavior of the dipole vortex after several oscillations. One might then expect the internal energy to be distributed in the most favorable way, and some regular behavior to appear.

Simulations have been done with parameter scans in $\beta$ and $\alpha$ in order to investigate the long time evolution of eastward propagating dipoles launched at the angle $\alpha$. The simulations are done with $a=1.0, U=0.1$, and $r_{\text {cut }}=3.5 a$.

Figure 6 shows trajectories for dipoles with $\beta=0.05$ and different initial angle. The most interesting feature is that they all relax to almost steady, eastward propagating dipoles, independently of the initial conditions. In this final state the distance between the two poles has increased with approximately $5 \%$, the propagation velocity has gone down to about $80 \%$ of the initial value, and the enstrophy has decreased by about $7 \%$.

For a large initial angle the trajectory is approximately sinusoidal, as one might expect. However, for smaller launch angles the initial part of the trajectory is rather irregular, indicating that the dipole in this phase is affected by the internal reorganization process.

Figure 7 shows trajectories for dipoles with $\alpha=30^{\circ}$ and different values of $\beta$. Again we observe the similarity in the long time behavior, independently of the initial conditions. Apparently the steady dipole propagating eastward is an attracting solution.

Simulations of dipoles with a very large initial launch angle are shown in Fig. 8. We observe that if the angle is too large, the dipole soon disintegrates. Thus there seems to be only two possible outcomes of a dipole vortex initially 


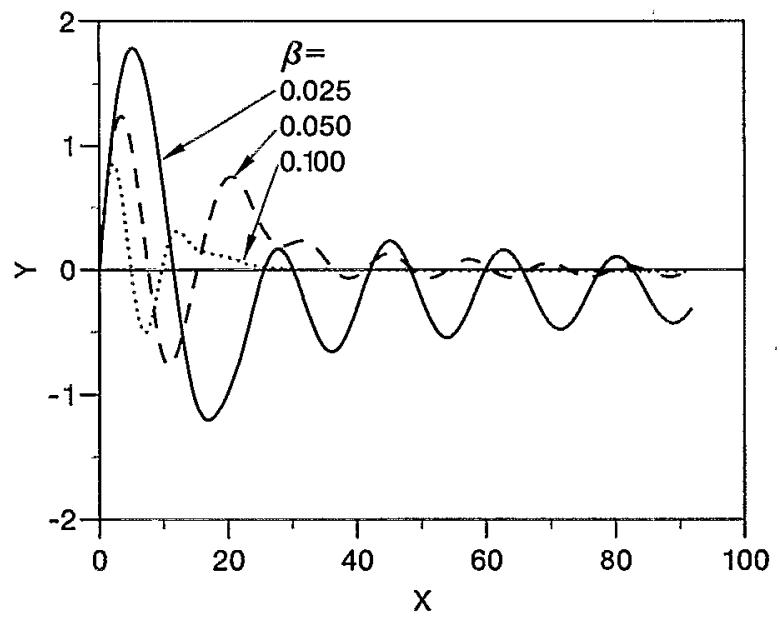

FIG. 7. Trajectories for initially eastward propagating dipole vortices; $\alpha=30^{\circ}$.

propagating at some angle. Either the dipole ends up as a steady eastward propagating dipole or it disintegrates. In order to examine the basin of attraction of the eastward propagating solution, we have plotted the result of a number of simulations on the $(\alpha, \beta / U)$ plane in Fig. 9. The simulations are done with $a=1.0, U=0.1, r_{\text {cut }}=3.5 a$. The maximum time for all simulations is $T_{\max }=400$, since it is observed that the disintegration happens before this time. (It is accelerated by the cutting.)

Simulations have also been done to investigate the long time evolution of westward propagating dipoles initially rotated by $\delta$ degrees $(\delta=180-\alpha)$ away from the negative $x$ axis. The simulations are done with $a=1.0, U=-0.1$, $\beta=0.05$, and $r_{\text {cut }}=4.0 a$ in the cutting facility.

Figure 10 clearly illustrates the linear instability of the westward propagating dipole, as discussed in the previous section. It is noteworthy that an initially westward propagating dipole may swing around and end up as an eastward propagating dipole. Comparing Fig. 6 and Fig. 10, it is also

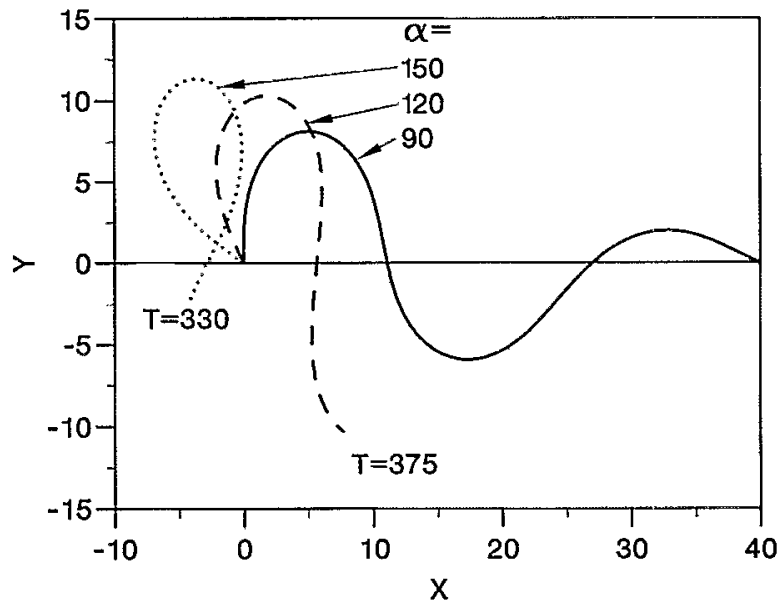

FIG. 8. Trajectories for initially eastward propagating dipole vortices. The indicated time is the approximate time of disintegration; $\beta=0.01$.

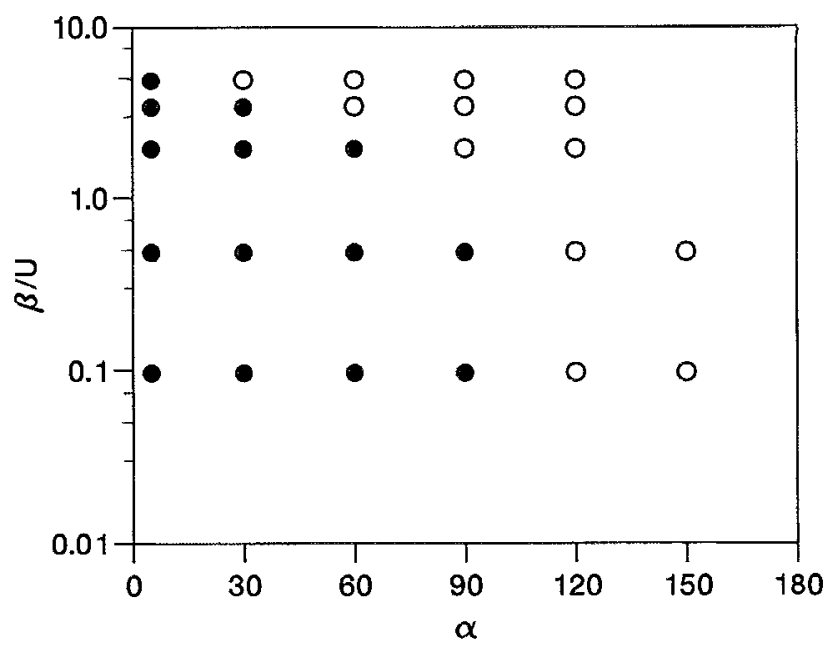

FIG. 9. Basin of attraction for initially eastward propagating dipole vortices. $O$ symbolized disintegrated dipoles, while symbolizes that in the long time limit the dipole vortex relax toward a steady eastward propagating dipole vortex. The result will be similar for negative angles due to the symmetry relation of Eq. (1).

evident that the westward propagating dipole is much less affected by the internal effects, which cause the irregular initial trajectories of the eastward propagating dipoles.

Similarly, as for the eastward propagating dipole vortex, we may calculate the ultimate fate of the tilted westward propagating dipole. This is shown in Fig. 11. The difference in the dynamical behavior of the two types of dipoles is possibly caused by the fact that the westward propagating dipole vortex is less localized than the eastward propagating dipole vortex. It was observed that in the limit of $\beta / U<1\left(\sim 10^{-2}-10^{-3}\right)$ the dynamical behavior of the perturbed dipoles is independent of the initial direction of propagation. This is not surprising, when it is remembered that in this limit $\rho \simeq 1$, and the structure is independent of the sign of $U$.

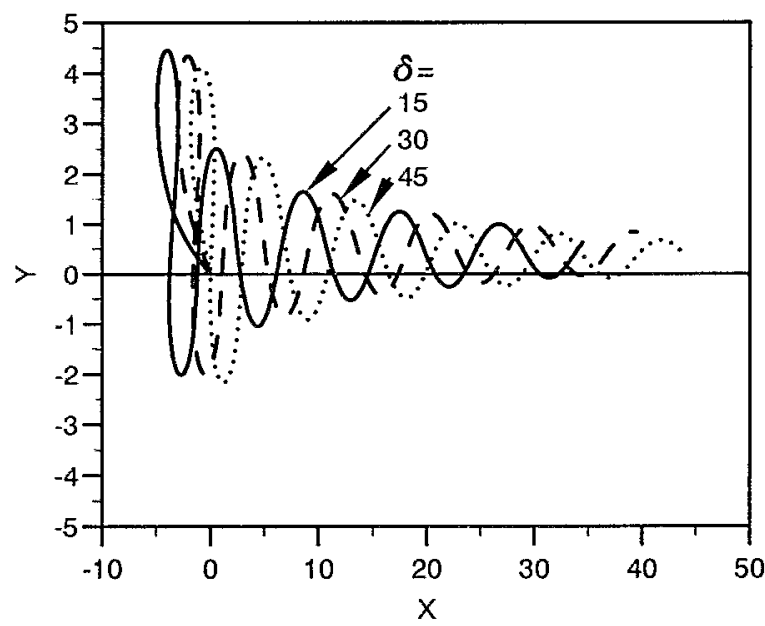

FIG. 10. Trajectories for initially westward propagating dipole vortices, i.e., $U$ is negative. Here $\delta \equiv 180-\alpha$ is the angle between the initial propagation direction and the negative $x$ axis; $\beta=0.05$. 


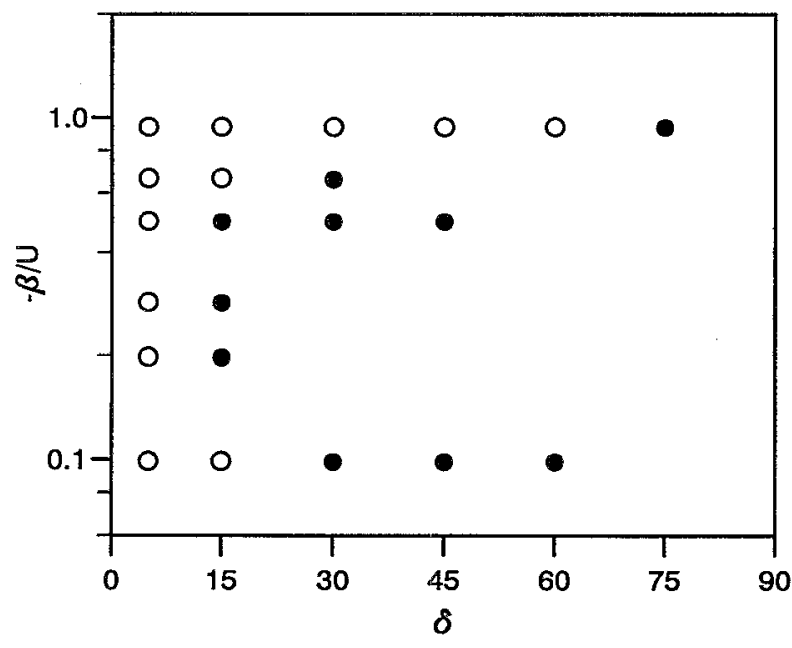

FIG. 11. Ultimate fate of initially westward propagating dipole vortices. Symbols as in Fig. 9.

\section{CONSEQUENCES OF ENSTROPHY LOSS}

As pointed out in Sec. II, the vortex gradually loses enstrophy to the surrounding fluid by nonviscous processes. This means that the amplitude $\phi-\nabla^{2} \phi$ must decrease in magnitude. Because of the conservation of potential vorticity, the cyclonic part must then move a little northward and the anticyclonic part a little southward. For a northeastward propagating dipole this induces a clockwise torque around the center of the dipole vortex and as a result we observe that the propagation angle decreases. Similar arguments may be adopted to explain why the westward propagating dipoles swing around and finally become eastward propagating. The enstrophy loss also causes the two dipole halves to be pulled slightly apart implying a small decrease in the propagation velocity. This corresponds well with the speculations used in Fig. 2 to explain the deviation between the Nycander-Isichenko ${ }^{14}$ theory and the simulations for $\beta / U>1$.

To express the effect of enstrophy loss on the propagation angle quantitatively, we calculate the change in enstrophy of the dipole vortex when it is rotated $\alpha$ degrees from exactly eastward, which is the position of minimum enstrophy. A first-order perturbation of Eq. (3) yields

$$
\delta \Omega=2 \int_{s} q \delta q d x d y=2 \beta \int_{s} q \delta y d x d y,
$$

where $q=\phi-\nabla^{2} \phi, \delta y$ is the vertical displacement of each fluid element due to the rotation, and the integral is taken over the surface inside the separatrix. Comparing with Eq. (9) we see that the right-hand side of Eq. (14) is just the change of the $y$ component of the dipole moment. Thus,

$$
\delta \Omega=2 \beta P_{d}^{\text {in }}(1-\cos \alpha),
$$

where $P_{d}^{\text {in }}$ is the dipole moment inside the separatrix. This expression is analogous to the change of the potential energy $(W)$ of an electrical dipole in an external homogeneous electrical field $\mathbf{E}$. For this dipole $W=-\mathbf{p} \cdot \mathbf{E}$, where $\mathbf{p}$ is the dipole moment of the electrical dipole.
Using that $P_{d}^{\text {in }} \sim(2 a)^{2} U S$ and that the total enstrophy is $\Omega_{0} \sim(2 a U)^{2} S$, the relative change of enstrophy is obtained

$$
\frac{\delta \Omega}{\Omega_{0}} \sim 2 \frac{\beta}{U}(1-\cos \alpha)
$$

This equation may be used to obtain a qualitative understanding of the unexpected deviation observed in Fig. 5, since it shows that in the limit of $\beta / U<1$ only minor relative changes in the enstrophy may cause a significant change of the propagation angle.

Simulations have been done to test Eq. (15). The cutting scheme selectively removes the enstrophy from the surrounding fluid (without it the code would conserve the total enstrophy of the flow). Since this fluid was initially quiescent, its enstrophy must originally have come from the dipole vortex. The cutting scheme can therefore be used for estimating the enstrophy lost by the dipole to the surrounding fluid. It was found that Eq. (15) overestimates the loss of enstrophy when $\alpha$ is large. However, results have been obtained indicating that large launch angles excite internal oscillations in the dipole vortex, with a frequency a few times larger than the oscillation frequency $\omega$ of the trajectory. This effect may account for the difference between Eq. (15) and the actual enstrophy loss, since the free energy obtained by decreasing the angle is partly lost to the surrounding fluid and partly used to excite the internal oscillations.

For small propagation angles Eq. (15) is found to underestimate the enstrophy loss, which we cannot explain. On the other hand, the loss is very small in this limit.

Nycander and Isichenko ${ }^{14}$ assumed that if the change of enstrophy was small compared to the total enstrophy of the dipole vortex, the dynamics would be unaltered. However, Eq. (15) shows that the characteristic enstrophy needed to affect the dynamics is of the order of $2 \beta P_{d}^{\mathrm{in}}$, which can be much smaller than $\Omega$. This explains why interactions between a dipole and low-amplitude linear waves may significantly affect the dipole trajectory as seen in Fig. 1. Similarly, we may expect that the enstrophy needed to split the dipole vortex into two vortices is of the order of $2 \beta P_{d}^{\text {in }}$, so that this quantity is analogous to a "binding energy."

\section{v. CONCLUSIONS}

It has been shown that the theory of Nycander and Isichenko ${ }^{14}$ describes the short time behavior of the oscillating dipole vortex quite well. However, since the dynamics of the nonstationary dipole is strongly affected by nonviscous loss of energy and enstrophy, the theory fails to describe the long time dynamics of the dipole vortex. It has also been shown that in part of the parameter space the short time vortex behavior is strongly affected by initial transient effects, caused by an internal reorganizing process.

In the long time limit dipoles launched at some finite angle are found to either end up as steady eastward propagating dipoles or disintegrate shortly after the initial pe- 
riod. Thus, the steady eastward propagating dipole vortex is an attracting solution. Our explanation of this is that eastward propagation is the minimum enstrophy state of the dipole vortex. The long time behavior can be interpreted as a relaxation toward this state, caused by enstrophy loss to the surrounding fluid. However, no definite quantitative confirmation of this has been obtained.

McWilliams ${ }^{7}$ and others ${ }^{18}$ have attempted to model atmospheric blocking, which is a midlatitude phenomenon characterized by unusual long time persistence of nearly stationary pressure cells, as a steady westward propagating dipole vortex. However, as has been seen in this work, the westward propagating dipole vortex is unstable and very sensitive to changes of enstrophy for small angles. The $e$-folding time of this tilt instability can be estimated theoretically as $2 a / \beta$, i.e., the diameter of the vortex divided by the maximum phase speed of Rossby waves, and in our simulations it is approximately two-thirds of this value. This is typically only a couple of days. (Verkley obtained 3.6 days for typical atmospheric parameters, but it should be remembered that the nature of his solution is different in that it contains a global pattern of standing waves.) The typical time scale of blocking events, on the other hand, is a few weeks. ${ }^{78,19}$ It is also clear that in order to observe dipole vortices in the atmosphere one should look for eastward propagating dipoles propagating at a speed being the sum of the dipole velocity and the mean westerly wind speed.

\section{ACKNOWLEDGMENTS}

The authors gratefully acknowledge many stimulating discussions with Dr. J. Juul Rasmussen, Risø National Laboratory. This work was performed while one of the authors (JN) was supported by a postdoctoral fellowship from the Swedish Natural Science Research Council. It is based on a M.Sc. dissertation ${ }^{20}$ by one of the authors (JSH), who is also thankful to Dr. O. Skovgaard and Dr. F. If from the Laboratory of Applied Mathematical Physics at the Technical University of Denmark for help and for providing computer facilities needed for the computational part of the present work.
${ }^{1}$ H. Lamb, Hydrodynamics, 6th ed. (Dover, New York, 1932).

${ }^{2}$ M. E. Stern, "Minimal properties of planetary eddies," J. Mar. Res. 33, 1 (1975).

${ }^{3}$ V. D. Larichev and G. M. Reznik, "Two-dimensional solitary Rossby waves," Dokl. Akad. Nauk SSSR 2331, 1077 (1976).

${ }^{4} \mathrm{~J}$. G. Charney, "The dynamics of long waves in a baroclinic westerly current," J. Meteorol. 4, 135 (1947).

${ }^{5}$ Y. Couder and C. Basdevant, "Experimental and numerical study of vortex couples in two-dimensional flows," J. Fluid Mech. 173, 225 (1986).

${ }^{6}$ G. R. Flierl, M. E. Stern, and J. A. Whitehead, "The physical significance of modons: Laboratory experiments and general integral constraints," Dyn. Atmos. Oceans 7, 233 (1983).

${ }^{7} \mathrm{~J}$. C. McWilliams, "An application of equivalent modons to atmospheric blocking," Dyn. Atmos. Oceans 5, 43 (1980).

${ }^{8}$ A. Hasegawa and K. Mima, "Pseudo-three-dimensional turbulence in magnetized nonuniform plasma," Phys. Fluids 21, 87 (1978).

${ }^{9}$ M. Makino, T. Kamimura, and T. Taniuti, "Dynamics of twodimensional solitary vortices in a low- $\beta$ plasma with convective motion," J. Phys. Soc. Jpn. 50, 980 (1981).

${ }^{10} \mathrm{~W}$. T. M. Verkley, "Stationary barotropic modons in westerly background flows," J. Atmos. Sci. 44, 2383 (1987).

${ }^{11}$ N. Y. Zabusky and J. C. McWilliams, "A modulated point-vortex model for geostrophic, $\beta$-plane dynamics," Phys. Fluids 25, 2175 (1982).

${ }^{12}$ D. D. Hobson, "A point vortex dipole model of an isolated modon," Phys. Fluids A 3, 3027 (1991).

${ }^{13} \mathrm{~J}$. Nycander, "Refutation of stability proofs for dipole vortices," Phys. Fluids A 4, 467 (1992).

${ }^{14} \mathrm{~J}$. Nycander and M. B. Isichenko, "Motion of dipole vortices in a weakly inhomogeneous medium and related convective transport," Phys. Fluids B 2, 2042 (1990).

${ }^{15}$ M. E. McIntyre and T. G. Shepherd, "An exact local conservation theorem for finite-amplitude disturbances to non-parallel Shear flows, with remarks on Hamiltonian structure and on Arnol'd's stability theorems," J. Fluid Mech. 181, 527 (1987).

${ }^{16} \mathrm{M}$. Kono and W. Horton, "Point vortex description of drift wave vortices: Dynamics and transport," Phys. Fluids B 3, 3255 (1991).

${ }^{17}$ G. F. Carnevale, G. K. Vallis, R. Porini, and M. Briscolini, "Propagation of barotropic modons over topography," Geophys. Astrophys. Fluid Dyn. 41, 45 (1988).

${ }^{18} \mathrm{~K}$. Haines, "Baroclinic modons as prototypes for atmospheric blocking," J. Atmos. Sci. 46, 3202 (1989).

${ }^{19} \mathrm{M}$. Mak, "Dynamics of an atmospheric blocking as deduced from its local energetics," Q. J. R. Meteorol. Soc. 117, 477 (1991).

${ }^{20} \mathrm{~J}$. S. Hesthaven, "Dynamics of coherent structures in the HasegawaMima equation," M.Sc. dissertation, Technical University of Denmark, 1991 (unpublished). 